\title{
Beitrag zur Morphogenese der epithelialen Hypophyse der Urodelen.
}

\author{
Von \\ Ryogen Sumi. \\ Aus dem Anatomischen Institut der Keio Universität, Tokyo. \\ Nit 7 Figuren im Text.
}

Die vorliegende Untersuchung wurde als ein Beitrag zu meiner vorigen Arbeit,") welche hauptsächlich die Entwicklung der Pars tuberalis der Hypophyse der in Japan einheimischen Urodelen (Hynobius und Onychodactylus) behandelte, weiterhin noch an anderen japanischen Urodelen, Diemictylus pyrrhogaster und Megalobatrachus maximus (Cryptobranchus japonicus) angestellt. Das Material wurde mit Formolwasser, und Formolalkohol fixiert. Die kleineren Objekte sind in Paraffin und die grösseren in Zelloidin eingebettet. Die Schnittdicke schwankt zwischen 10-30 $M$ (10-20 $M$ bei Paraffineinbettung, $30 M$ bei Zelloidineinbettung). Die Färbung wurde mit Hämatoxylin-Eosin und Eisenhämatoxylin nach Weigert (Stückfärbung) ausgeführt. Aus 5 Schnittserien wurden 5 Modelle in bekannter Weise nach dem Bornschen Verfahren angefertigt.

\section{Eigene Befunde.}

\section{Diemictylus.}

\section{Stadium 1.}

$15 \mathrm{~mm}$ lange Larve (die Vorderglieder sind gut erkennbar, die hinteren dagegen .noch nicht angelegt).

In diesem Stadium ist das Infundibulum, das der ventralen Seite des Mesencephalon dicht anliegt, bereits gut entfaltet und liegt im hinteren Teil der Fenestra basicranii. Die Hypophyse bedeckt die ventrale und kaudale Partie des Infundibulums und ihr kaudaler Teil

1) Sumi, R., On the morphogenesis of the epithelial hypophysis of the tailed amphibia. Folia anat. jap. Bd. II, 1924. 
liegt dicht der dorsalen Fläche der Chorda dorsalis an (Fig. 2). Hier kann man die Pars tuberalis noch nicht vorfinden. Die Hypophyse stellt von ventral gesehen eine dreieckige Form dar, deren vorderes Ende breit, deren hinteres dagegen eng ist (Fig. 1). Auf dem Querschnitt sieht man, dass der vordere Teil der Hypophyse sich dicht der ziemlich entfalteten ventralen

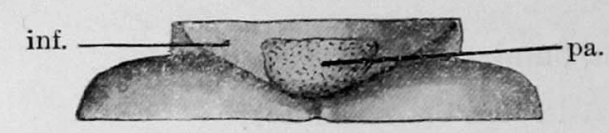

Fig. 1.

Ventrale Ansicht des Wachsplattenmodels der Hypophyse. $15 \mathrm{~mm}$ lange Larve, $\times 50$. inf. Infundbulum, pa. Pars anterior.

Wand des Infundibulums anliegt und es sieht aus, als ob er einen Nebenteil des letzteren bilde. Während die Infundibularwand am kaudalen Teil stark verdünnt

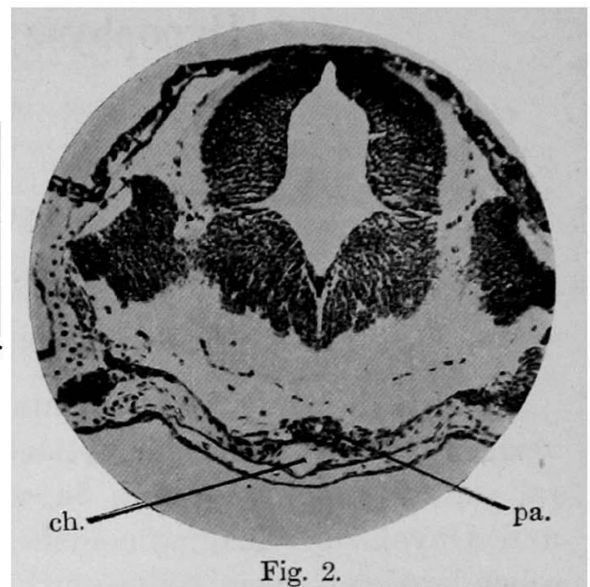

Querschnitt durch den kaudalen Teil der Hypophyse. $15 \mathrm{~mm}$ lange Larve. ch. Chorda, pa. Pars anterior.

ist, ist die Hypophyse dagegen an Grösse vermehrt und hilft in den Infundibularventrikel eindringend die ventrale Infundibularwand $\mathrm{zu}$ bilden.

\section{Stadium 2.}

$32 \mathrm{~mm}$ lange Larve (die linken Kiemenbüschel sind noch vorhanden, die rechten völlig verschwunden. Die Vorder- und Hinterglieder sind gut entwickelt).

In diesem Stadium liegt das Infundibulum in dem hinteren Teil der Fenestra basicranii, aber ziemlich entfernt von der Chorda dorsalis und dem Planum basale. Die Hypophyse ist an ihrer transversalen Breite und rostrokaudalen Länge gegenüber dem vorigen Stadium bedeutend vermehrt, bedeckt das Infundibulum von ventral und kaudal und zeigt bei ventraler Ansicht eine Trapezform, die kaudal breit und rostral schmal ist (Fig. 3). Sie zeigt hier, wie beim vorigen Stadium, je nach dem vorderen und hinteren Teil ein ganz anderes Aussehen. Die ventrale Infundibulumwand ist am kranialen Teil verdickt und dadurch sieht die Hypophyse aus, als ob sie dessen Nebengebilde sei, während sie am kaudalen Teil dorsoventral dicker ist und in den Infundibularventrikel eindringend sich an der Bildung der ventralen Wand des Infundibu- 
lums beteiligt. Die Verbindung zwischen der Hypophyse und der ventralen Wand des Infundibulums ist sehr innig, aber man kann die Grenze

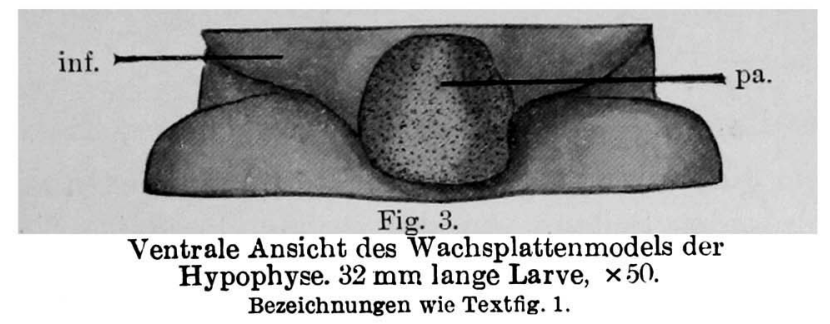

zwischen beiden klar wahrnehmen, während die Hypophyse ventral mit , einer bindegewebigen Membran, die an der Fenestra basicranii ausgespannt ist, im ganz lockeren Zusammenhang steht.

\section{Stadium 3.}

$34 \mathrm{~mm}$ langes Tier kurz nach der Metamorphose, die beiderseitigen Kiemen sind bereits verloren gegangen.

In diesem Stadium verhält sich im allgemeinen die Hypophyse gleich wie beim vorigen Stadium, liegt jedoch etwas weiter entfernt von der Chorda dorsalis und dem Planum basale und verbindet sich auch locker mit der ventralen bindegewebigen Membran. Das Infundibulum liegt ziemlich weit entfernt von der ventralen Seite des Mesencephalon. Bemerkenswert ist in diesem Stadium das erste Auftreten der Pars tuberalis (Fig. 4). So springen die beiderseitigen Partes tuberales ganz

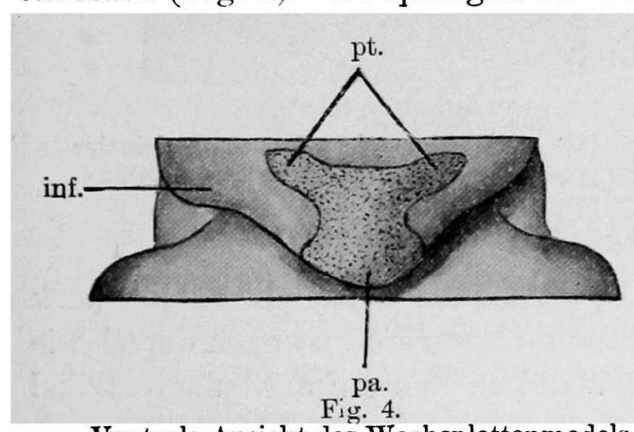

Ventrale Ansicht des Wachsplattenmodels der Hypophyse. $34 \mathrm{~mm}$ lange Larve, $\times 50$. pa. Pars anterior, pt. Pars tuberalis. inf. Infundibulum.
inturion symmetrisch aus den beiderseitigen rostrolateralen Ecken der Pars anterior mit breiter Basis schief nach laterorostral hervor und liegen dicht an der ventralen Wand des Infundibulums. Die Pars anterior bedeckt am kranialen Teil das Infundibulum von ventral her, indem sie eine Art Anhangsgebilde des Infundibulums darstellt. Das Infundibulum wird nach kaudal immer mehr dünner, während die Pars anterior dagegen immer dicker wird. Also wölbt diese sich zwischen den beiderseitigen Seitenwülsten vor, als ob die letzteren sie von beiden Seiten zu umgreifen scheinen. Man kann jedoch die Grenze zwischen diesen beiden gut erkennen. Bei 
ventraler Ansicht stellt die Pars anterior eine dreieckige Form dar, deren schmale Spitze nach kaudal gerichtet ist. Der Mittelteil der Hypophyse ist eingeschnürt und dadurch sieht die letztere in zwei Lappen eingeteilt aus.

Stadium 4. $135 \mathrm{~mm}$ langes ausgewachsenes Tier.

In diesem Stadium ist auffallend die Formverschiedenheit der Pars tuberalis nach beiden Seiten. An der rechten Seite ist die Pars vollständig isoliert und steht $0.75 \mathrm{~mm}$ entfernt vom vorderen Rand der Pars anterior, während sie an der linkén mit einer breiten Basis mit der rostrolateralen Ecke der letzteren sich verbindet und ihre transversale Breite vom Anfang bis zum Ende als die gleiche bleibt (Fig. 5).

Die beiderseitigen Partes tuberales legen sich dicht der ventralen Wand des Infundibulums an. Die Längsachsen der beiderseitigen Partes laufen von kaudomedial nach rostrolateral, so kreuzen sich ihre kaudalen Verlängerungen etwa im vorderen Teil der Pars anterior miteinander. Die rechte Pars liegt weiter nach rostral verschoben als die linke. Aus diesem Befunde kaun man vermuten, dass die rechte Pars tuberalis auch früher mit der Pars anterior verbunden war. Die transversale Breite der ovalen Pars anterior ist am Mittelteil am grössten, während ihr vorderes und hinteres Ende verschmälert sind (Fig. 5). Die beiden lateralen Sei-

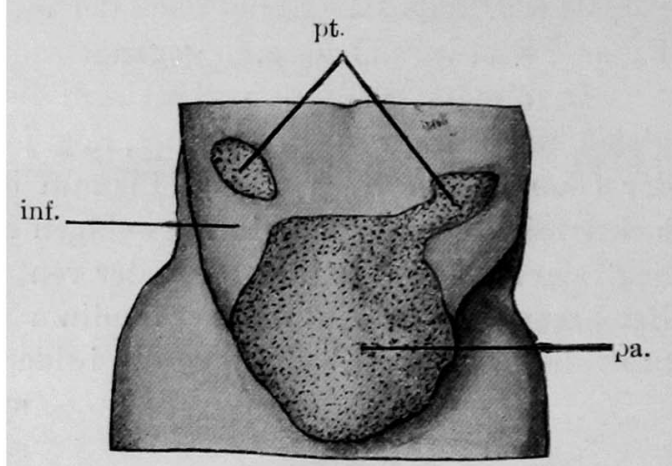
Fig. 5.

Ventrale Ansicht des Wachsplattenmodels der Hypophyse. $135 \mathrm{~mm}$ langes ausgewachsenes Tier, $\times 33$ Bezeichnungen wie Textfig. 4. ten der Hypophyse verbinden sich in der kaudalen Gegend mit der stark verdünnten Infundibularwand und ihr mittlerer Teil ist nach dorsal wie ventral konvex, so dass ihre dorsale Wand in den Infundibularventrikel eindringend die ventrale Wand des Infundibulums zu bilden hilft.

Stadium 5. $140 \mathrm{~mm}$ langes ausgewachsenes 'Tier.

In diesem Stadium sind die beiderseitigen Partes tuberales von der Pars anterior vollständig getrennt, die rechte liegt $0.12 \mathrm{~mm}$ weit entfernt von der letzteren und die linke $0.15 \mathrm{~mm}$ davon. Die Längsachsen der beiderseitigen Partes tuberales laufen schief von kaudomedial nach 
rostrolateral, so kreuzen sich ihre kaudalen Verlängerungen etwa im mittleren Teil der Pars anterior miteinander (Fig. 7). Diese Befunde

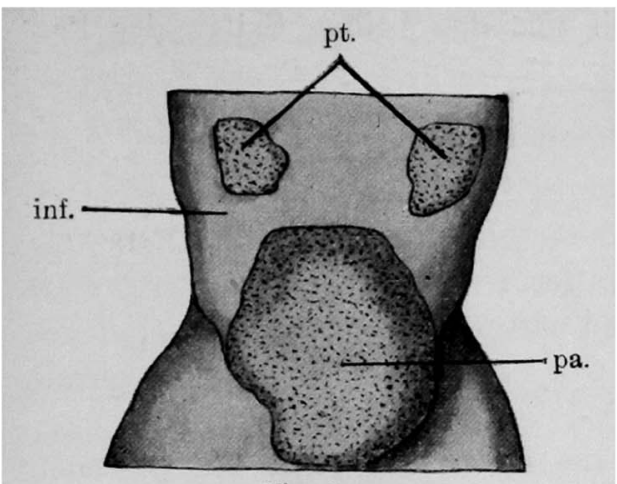

Fig. 6.

Ventrale Ansicht des Wachsplattenmodels der Hypophyse. $140 \mathrm{~mm}$ langes ausgewachsenes Tier, $\times 33$. Bezeichnungen wie Textfig. 4. weisen darauf hin, dass die beiderseitigen Partes tuberales früher mit der Pars anterior verbunden waren, wie oben erwähnt. Die beiderseitigen legen sich dicht der ventralen Wand des Infundibulums an, die rechte ist etwas kleiner als die linke (Fig. 6).

Die Pars anterior, die hier sehr voluminös geworden ist, bedeckt das Infundibulum von ventral und kaudal her und hat ihren transversalen wie rostrokaudalen Durchmesser vermehrt. Ihre Form ist oval, indem der Mittelteil am breitesten ist (Fig. 6). Die

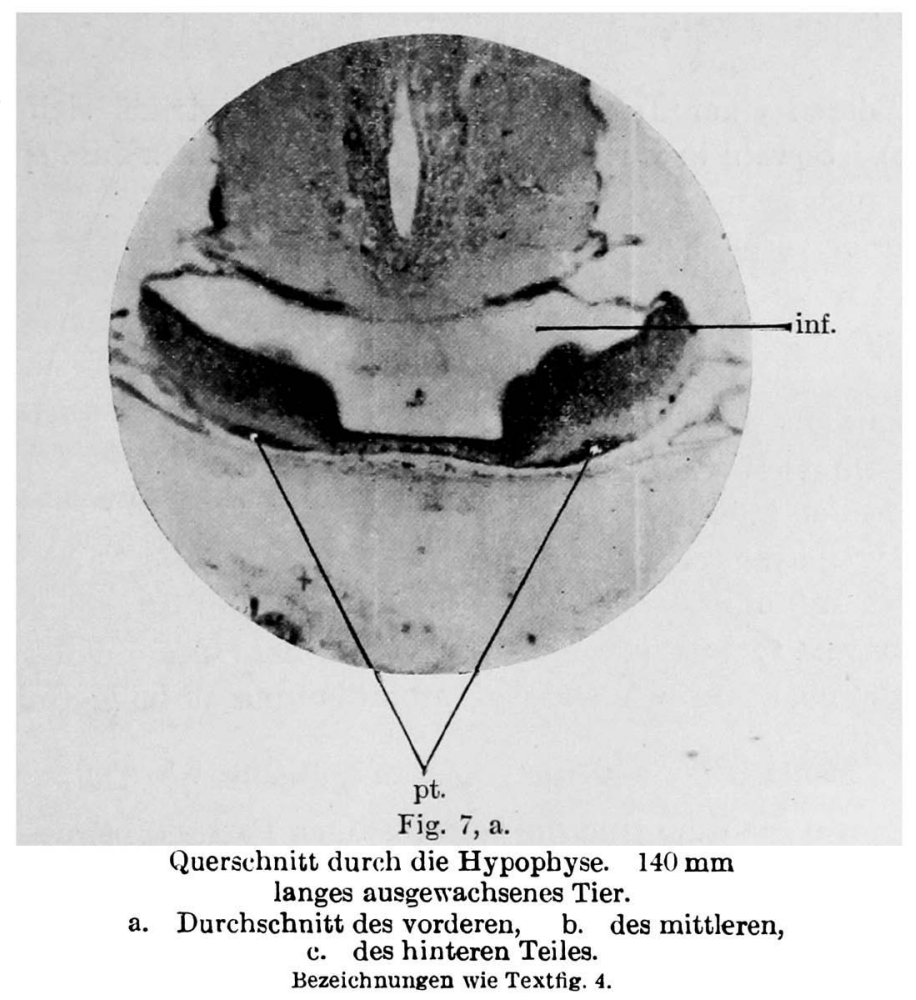


Verhältnisse zwischen ihr und der Infundibularwand sind dieselben wie beim vorigen Stadium; so ist die Infundibularwand in der Kaudalgegend stark verdünnt und verbindet sich mit den beiden Seiten der Pars

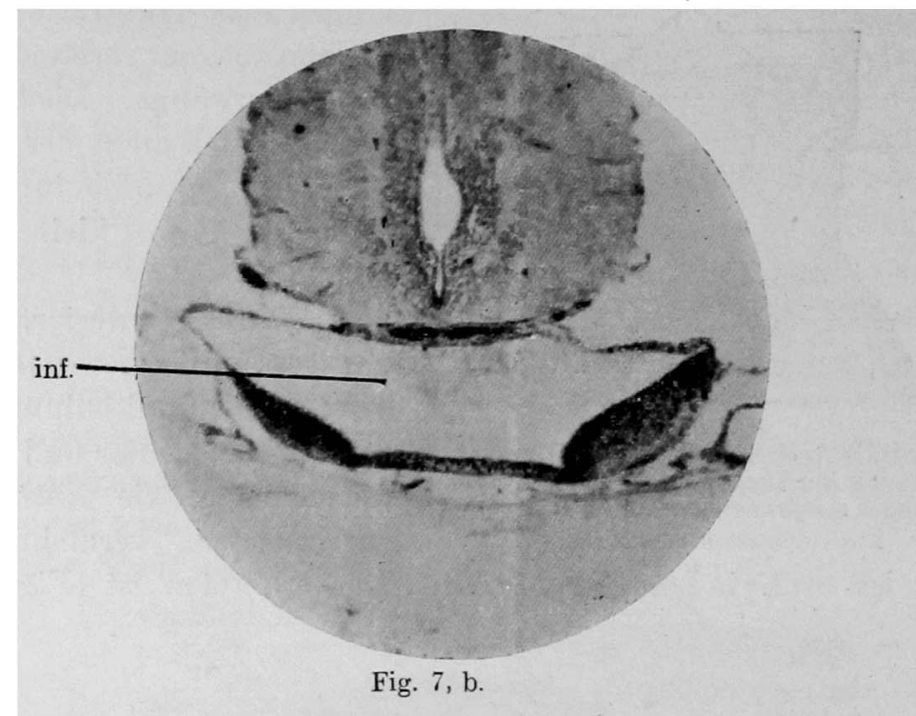

anterior, deren einer Teil in den Infundibularventrikel sich vorwölbt (Fig. 7, c). Sowohl in vorigem als auch in diesem Stadium springt das

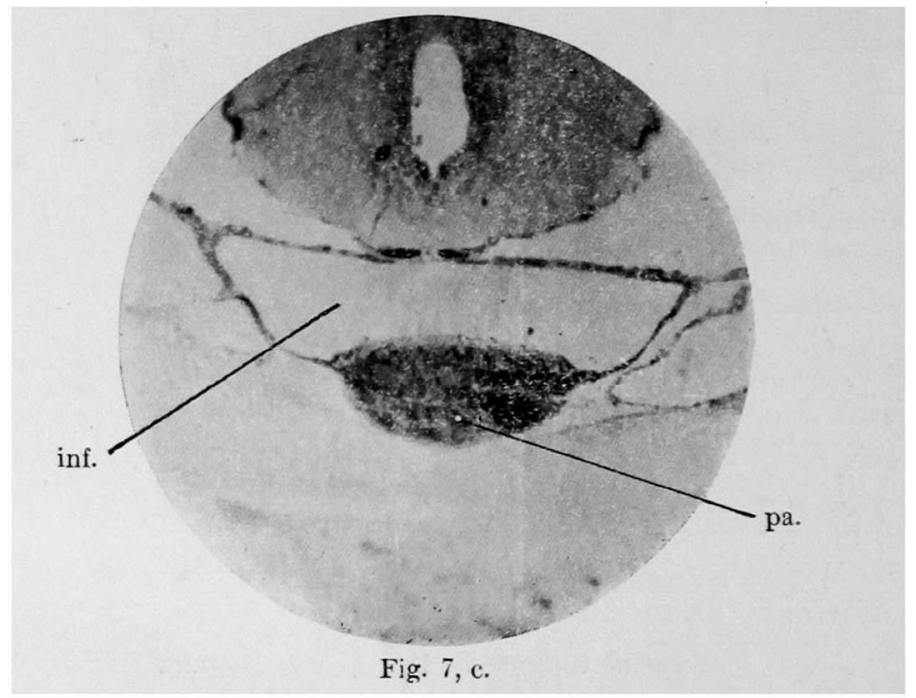

Infundibulum aus der ventralen Seite des Diencephalon hervor und läuft schief von kraniodorsal nach kaudoventral, so dass sein kaudales Ende 
weiter als das kraniale von der ventralen Wand des Mesencephalon, auch als bei den larvalen Stadien, entfernt liegt.

Bei den anderen 7 untersuchten Larven war die Pars tuberalis nicht vorhanden, während bei 13 ausgewachsenen Exemplaren, mit Ausnahme von 2 Fällen, stets ihr Vorhandensein konstatiert worden ist.

\section{Megalobatrachus maximus.}

Beim Megalobatrachus maximus konnte ich leider kein larvales Stadium verfolgen. Bej 3 von mir untersuchten ausgewachsenen Exemplaren war die Pars tuberalis niernals vorhanden.

\section{Vergleichung und Zusammenstellung.}

1. Pars anterior.

Die Pars anterior stellt den Hauptteil der Hypophyse dar und bedeckt das Infundibulum von ventral und kaudal her. Sie ist aber bis zur Zeit der Metamorphose nicht so stark entfaltet, während sie bei ausgewachsenen Tiere sehr deutlich an Volum vermehrt ist und ein ansehnliches Gebilde an der ventralen Seite des Infundibulums darstellt. Ihre Form ist je nach den Stadien und Individuen sehr verschieden, aber sie ist im allgemeinen oval. Sie liegt in der Fenestra basicranii, aber ihre Lage ist auch je nach den Stadien sehr verschieden. In den früheren Stadien legt sie sich der dorsalen Seite der Chorda dorsalis dicht an, aber in den vorgerückten Stadien verschiebt sie sich immer mehr rostralwärts und kommt sehr entfernt von der Chorda dorsalis und dem Planum basale zu stehen. Diese Verschiebung der Pars anterior wird wahrscheinlich bedingt durch den fortschreitenden Entwicklungsvorgang des Gehirns, nämlich durch die Erstreckung der Gehirnkrümmungen.

Am rostralen 'T'eil liegt die Pars anterior wie ein Anhangsgebilde ventral vom Infundibulum, dessen ventrale Wand hier ziemlich verdickt ist, während am kaudalen Teil ihre dorsale Partie mit der verdünnten ventralen Wand des Infundibulums zusammen in den Infundibularventrikel eindringt. Diese Pars verbindet sich anderseits locker mit einer bindegewebigen Membran, die sich an der Fenestra basicranii ausspannt.

Im folgenden gebe ich tabellarisch die Grösse der Pars anterior, die erst an Modellen gemessen und dann in die natürlichen Massen umgerechnet ist, an. 
TABELLE I.

\begin{tabular}{|c|c|c|c|c|c|c|}
\hline & Stadium & I & ]I & III & IV & V \\
\hline & Körperlänge (mm) & 15 & 32 & 34 & 135 & 140 \\
\hline & $\begin{array}{l}\text { Mediane rostrokaudale } \\
\text { Länge der Pars anterior } \\
(\mathrm{mm})\end{array}$ & 0.14 & 0.30 & 0.28 & 0.90 & 0.89 \\
\hline \multirow{3}{*}{ 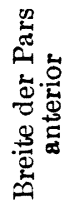 } & Vorderteil (mm) & 0.23 & 0.16 & 0.32 & 0.60 & 0.46 \\
\hline & Mittelteil (mm) & 0.22 & 0.32 & $* 0.22$ & 0.99 & 0.75 \\
\hline & Hinterteil (mm) & 0.14 & 0.36 & 0.14 & 0.36 & 0.33 \\
\hline
\end{tabular}

* Die geringste Breite des Mittelteils.

2. Pars tuberalis

Die Pars tuberalis bildet die rostrale Fortsetzung der Pars anterior. Beim Diemictylus springt sie aus der rostrolateralen Ecke der letzteren vor und ist von kaudomedial nach rostrolateral zu gerichtet. Die Verlängerungen der Längsachsen der beiderseitigen Partes schneiden sich im rostralen Teil der Pars anterior sowohl im getrennten als auch im verbundenen Typus.

Das erste Auftreten der Pars tuberalis beim Diemictylus findet sowohl in larvalen Stadien als auch nach der Metamorphose statt. Im allgemeinen kornmt sie kurz nach oder vor der Metamorphose zum Vorschein (Tabelle V). Die Trennung der Pars tuberalis von der Pars anterior geschieht erst nach der Metamorphose, während ich vor der Metamorphose keine solche bemerken konnte. Der Zeitraum von der Metamorphose bis zur Trennung ist sehr verschieden. In den ausgewachsenen Stadien findet man zwei Typen der Pars tuberalis, von denen der eine den getrennten Typus darstellt, dagegen der andere den verbundenen. Im letzteren Typus war jedoch nur die einseitige, nämlich die linke Pars tuberalis mit der Pars anterior verbunden. Daraus kann man sagen, dass die Pars tuberalis von der Pars anterior stets nach der Metamorphose erst an der rechten Seite, dann an der linken sich trennt und in den ausgewachsenen Stadien bleibt öfters der einen Seite mit der Pars anterior verbunden. Hier kann ich jedoch nicht entscheidend sagen, ob die Trennung weit oder kurz nach der Metamorphose geschieht, da ich kein geeignetes Material gehabt habe, um die zwischen dem 
Stadium 3 und 4 sich abspielenden Vorgänge zu erkennen. Ich bin der Meinung, dass zwischen der Metamorphose und der Trennung der Pars tuberalis kein funktioneller Zusammenhang besteht, während Atwell ${ }^{1)}$ bei Anuren folgendes behauptet. "The further development of the pars tuberalis consists in a spreading out of the lateral lobes under the brain floor and their subsequent detachment from the anterior lobe proper. This latter occurs in Rana clamitans and Bufo americana at least, during the latter part of metamorphosis or very soon after ist completion."

Nach meiner Meinung geschieht die Trennung der Pars tuberalis von der Pars anterior nicht als die Folge der funktionellen Veränderung bei der Metamorphose, vielmehr wird die Verlagerung durch die Formveränderung des Infundibulums verursacht. So möchte ich sagen, dass die Pars tuberalis nur ganz durch die morphologische Entwicklungsmechanik gebildet wird. Die Hypophysenanlage entsteht anfangs aus Ektoderm und kommt kurz unter dem Infundibulum. Danach übertrifft die Entwicklung des Infundibulums die der Hypophyse und die letztere wird durch das erstere nach kaudal gedrängt und kommt an dessen kaudalem Ende zu liegen. Dabei entwickelt sich die Hypophyse gleichzeitig so stark in einer kleinen Kranialhöhle, dass ihre kleinen lateralen Partien an den lateralen Seiten des Infundibulums vorspringen und die Partes tuberales bilden. Daher ist es mir wahrscheinlich, dass die Pars tuberalis nicht nur durch den rostralen Zuwachs der Pars anterior gebildet wird, wie Atwell ('18) sagt, sondern auch die starke Entwicklung sowohl der Hypophyse als auch des Infundibulums dabei helfen. Nachher zeigt das Infundibulum die Neigung, immer mehr sich nach ventralwärts zu richten, wodurch die Pars tuberalis von der Pars anterior abgetrennt wird.

In Bezug auf das Fehlen der Pars tuberalis sowohl beim Diemictylus als auch beim Megalobatrachus kann man an zwei Möglichkeiten denken, dass einmal die Pars tuberalis von Anfang an nicht angelegt ist und anderseits dass sie im Entwicklungsverlaufe verschwindet. Ich kann augenblicklich nicht mit Sicherheit entscheiden, welche von den beiden zutrifft. Meiner Meinung nach wäre es jedoch richtiger zu denken, dass das Fehlen der Pars tuberalis nur beim Diemictylus im Verlaufe der Entwicklung zu stande kommt.

Ich möchte aus Atwells's) ('18, '21) und meinen eigenen Ergeb-

1) Atwell, W. J., The development of the hypophysis of the anura. Anat. Rec. Vol. 15. 1918.

2) Atwell, W. J., The morphogenesis of the hypophysis in the tailed amphibia. Anat. Rec. Vol. 22. 1921. 
Ryogen Sumi,

nissen an der Hypophyse der Amphibien folgende 3 Typen unterscheiden : U-Typus, Ö-Typus und O-Typus. Beim U-Typus springen die beiderseitigen Partes tuberales von den beiderseitigen vorderen lateralen Ecken der Pars anterior, beim Ö-Typus liegen sie von der Pars anterior getrennt und beim O-Typus sind sie wesentlich nicht vorhanden. Alle diese I'ypen können natürlich die Übergangsform zwischen sich haben. Beim Diemictylus kommt zuweilen eine Übergangsform zwischen U-und Ö-Typus vor, indem die Pars tuberalis nur an einer Seite mit der Pars anterior verbunden ist. Als eine Unterart des U-Typus, wo die beiden Partes tuberales miteinander parallel ziehend von den beiderseitigen vordelen lateralen Ecken vorspringen, möchte ich noch den $\vee$-Typus angeben, bei dem die nach hinten konvergierend, in der Nähe der medianen vorderen Seite der Pars anterior inserierenden beiden Partes tuberales vorkommen. Ein O-Typus, der die Übergangsform zwischen dem Ö-und O-Typus darstellen könnte, liess sich ganz und gar nicht konstatieren.

TABELLE II.

\begin{tabular}{|c|c|c|c|c|c|}
\hline & & U-Typus & $\downarrow$-Турия & O-Typus & O-Typus \\
\hline Anuren & $\begin{array}{l}\text { Larve } \\
\text { Ausgewachsene }\end{array}$ & \pm & $\overline{-}$ & $\overline{+}$ & $\overline{-}$ \\
\hline Diemictylus & $\begin{array}{l}\text { Larve } \\
\text { Ausgewachsene }\end{array}$ & $\begin{array}{l}+ \\
+\end{array}$ & $\overline{-}$ & $\overline{+}$ & $\bar{t}$ \\
\hline Onychodactylus & $\begin{array}{l}\text { Larve } \\
\text { Ausgewachsene }\end{array}$ & \pm & $\overline{+}$ & $\overline{-}$ & $\overline{-}$ \\
\hline Hynobius & $\begin{array}{l}\text { Larve } \\
\text { Ausgewachsene }\end{array}$ & \pm & $\overline{+}$ & $\overline{-}$ & $\overline{-}$ \\
\hline Necturus & $\begin{array}{l}\text { Larve } \\
\text { Ausgewachsene }\end{array}$ & $\overline{+}$ & $\overline{-}$ & $\overline{-}$ & $\overline{-}$ \\
\hline Amblystoma & $\begin{array}{l}\text { Larve } \\
\text { Ausgewachsene }\end{array}$ & + & $\overline{-}$ & $\overline{-}$ & $\overline{-}$ \\
\hline Amphiuma & $\begin{array}{l}\text { Larve } \\
\text { Ausgewachsene }\end{array}$ & $\overline{+}$ & - & $\overline{-}$ & $\overline{-}$ \\
\hline Megalobatrachus & $\begin{array}{l}\text { Larve } \\
\text { Ausgewachsene }\end{array}$ & $\overline{-}$ & $\overline{-}$ & $\overline{-}$ & $\overline{+}$ \\
\hline
\end{tabular}

- = nicht vorhanden.

$+=$ vorhanden.

Die Grösse der Pars tuberalis, die an Modellen gemessen und in die natürliche Grösse umgerechnet wurde, ist folgendermassen. 
Tabelle III.

\begin{tabular}{|c|c|c|c|c|}
\hline & \multicolumn{2}{|c|}{ Stadium IV } & \multicolumn{2}{c|}{ Stadium V } \\
\hline & Rechts & Links & Rechts & Links \\
\hline $\begin{array}{c}\text { Rostrokaudale } \\
\text { Länge (mm) }\end{array}$ & 0.30 & 0.30 & 0.21 & 0.27 \\
\hline $\begin{array}{c}\text { Transversale } \\
\text { Breite (mm) }\end{array}$ & 0.27 & 0.30 & 0.21 & 0.24 \\
\hline
\end{tabular}

Bei den ausgewachsenen Exemplaren verhält sich der Zusammenhang zwischen der Pars tuberalis und anterior folgendermassen.

Tabelle IV-

\begin{tabular}{|c|c|c|c|}
\hline $\begin{array}{c}\text { Beiderseitige } \\
\text { Verbindung }\end{array}$ & $\begin{array}{c}\text { Beiderseitige } \\
\text { Trennung }\end{array}$ & $\begin{array}{c}\text { Einseitige } \\
\text { Trennung }\end{array}$ & $\begin{array}{c}\text { Ohne Pars } \\
\text { tuberalis }\end{array}$ \\
\hline $2(15 \%)$ & $7(53 \%)$ & $2(15 \%)$ & $2(15 \%)$ \\
\hline
\end{tabular}

Das Vorhandensein der Pars tuberalis nach oder vor der Metamorphose verhält sich folgendermassen.

Tabelle V.

\begin{tabular}{|l|c|c|c|c|c|c|c|c|c|c|c|}
\hline Körperlänge (mm) & 9 & 11 & 13 & 15 & 15 & 17 & 28 & 32 & 33 & $* 33$ & $* 33$ \\
\hline & - & - & - & - & - & - & - & - & + & + & + \\
\hline
\end{tabular}

$-=$ nicht vorhanden.

$+=$ vorhanden.

* = kurz nach der Metamorphose.

\section{Zusammenfassung.}

1. Pars anterior und tuberalis beim Diemictylus liegen beide der ventralen Seite des Infundibulums dicht an. Die Pars anterior ist rostral dünn, dagegen kaudal sehr verdickt, dringt in den Infundibularventrikel ein und hilft die ventrale Wand des Infundibulums zu bilden.

2. Das erste Auftreten der Pars tuberalis findet sowohl vor als auch nach der Metamorphose statt, aber stets in zeitlicher Nähe der Metamorphose.

3. Die Isolierung der Pars tuberalis findet stets nach der Metamorphose statt. Nach meiner Meinung geschieht ihre Isolierung unabhängig 
von der funktionnellen Wirkung bei der Metamorphose, sie wird vielmehr nur durch die Wachstumsmechanik des Infundibulums und der Hypophrse verursacht.

4. Bei den Amphibien unterscheide ich nach den Lageverhälnissen der Pars tuberalis zur Hypophyse 3 Typen an dieser letzteren; nämlich L'-Tỵpus, Ö-Typus und O-Typus.

5. Die Hypophyse des Diemictylus stellt in Bezug auf die Lage der Pars tuberalis den Übergangstypas zwischen den Anuręn- und Urodelentypen dar.

6. Die Trennung der Pars tuberalis beginnt stets erst auf der rechten Seite.

7. Die Längsachsen der beiderseitigen Partes tuberales konvergieren nach hinten mehr oder weniger gegeneinander.

8. Beim Diemictylus fehlt die Pars tuberalis zuweilen (15\%) beiderseitig rollständig.

9. Am ausgewachsenen Megalobatrachus fehlt stets eine Pars tuberalis.

Zum Schluss möchte ich Herrn Prof. Okajima für seine freundliche Unterstützung meinen herzlichen Dank aussprechen. 